\title{
Resident Perceptions of Anatomy Education: A Survey of Medical School Alumni From Two Different Anatomy Curricula and Multiple Medical Specialties
}

\author{
Michael A. Bohl, ${ }^{\star}$ Thomas R. Gest \\ Division of Anatomical Sciences, University of Michigan Medical School, Ann Arbor, Michigan
}

\begin{abstract}
In 2004, the University of Michigan Medical School reduced its gross anatomy curriculum. To determine the effect of this reduction on resident perceptions of their clinical preparedness, we surveyed alumni that included residents from the original and new shortened curricula. A Likert-scale survey was sent to four classes of alumni. Respondents were compared in old curriculum (OC) and new curriculum (NC) groups, surgical specialty (SS) and nonsurgical specialty (NS) groups, and subgroups of SS and NS were compared for differences between OC and NC. Mean response scores were compared using independent samples $T$-tests. As a single population $(n=110)$, respondents felt their anatomy education prepared them well for residency, that a more robust anatomy curriculum would be helpful, that dissection was important to their residency preparation, and that a 4th year anatomy elective was effective in expanding their anatomy education and preparing them for residency. No significant difference existed between OC and NC groups, neither as a whole nor as SS and NS subgroups. The SS group felt dissection was more important to their residency preparation than the NS group $(P=0.001)$ and that a more robust anatomy curriculum would have better prepared them for residency $(P=0.001)$. Thirty percent of SS respondents who did not take a 4th year elective commented that they wish they had. Fourth year anatomy electives were highly valued by residents, and respondents felt that they should be offered to students as a way of revisiting anatomy following the 1st year of clinical training. Anat Sci Educ 4:126-131. @ 2011 American Association of Anatomists.
\end{abstract}

Key words: effectiveness of anatomy education; gross anatomy education; undergraduate medical education; clinical preparedness; fourth year anatomy electives; medical school alumni

\section{INTRODUCTION}

Anatomical sciences appear to be taking a diminishing role in undergraduate medical education. Gartner (2003) shows that total curriculum hours devoted to gross anatomy in United States medical schools dropped from an average of 249 hours

*Correspondence to: Mr. Michael A. Bohl, Division of Anatomical Sciences, University of Michigan Medical School, Ann Arbor, MI 48109-0608, USA. E-mail: mbohl@umich.edu

Received 13 September 2010; Revised 10 January 2011; Accepted 11 January 2011.

Published online 4 March 2011 in Wiley Online Library (wileyonlinelibrary.com). DOI 10.1002/ase.207

(C) 2011 American Association of Anatomists to 144 hours between 1967 and 2001, and Drake et al. (2009) show that this trend has continued internationally over the last decade. Some believe the driving force behind this reduction has been the exponential increase in medical knowledge that has occurred during this period, creating a body of medical science knowledge that is too large to be practically taught or learned in an undergraduate medical curriculum (Bergman et al., 2008). The solution to this dilemma sought by many medical schools has been to triage the basic sciences, focusing primarily on what is foundational for future learning and eliminating that which is deemed excessively factual or less clinically relevant. The General Medical Council of the United Kingdom, for example, issued a statement in 1993 to its medical schools that "undue emphasis on detail was not required" and that the "burden" of factual information should be reduced (Patel and Moxham, 2008). As a subject that is considered by many in medical 
school administration to be excessively factual and less clinically important, anatomy is often targeted when medical schools are looking to create more space in their curricula. There is also a significant financial motivation to reductions in anatomy education as it usually requires expensive laboratory space, faculty time, and cadaver expenses.

Some believe that although the academic community has become desensitized to the concerned voices of senior anatomists and surgeons arguing for the preservation of anatomy education, perhaps concerns raised by medical studentsthose with first hand experience of the current system-may be harder to ignore (Gogalniceanu et al., 2010). Surveys from the last decade on medical student opinion of their anatomy education indicate that the majority of medical students and professors feel further cuts in gross anatomy and cadaver dissection should be avoided (Leong, 1999; Patel and Moxham, 2008; Holla et al., 2009; Gogalniceanu et al., 2010). These studies show that both students and faculty believe cadaver dissection is the centerpiece of gross anatomy study and that the anatomy they are learning is clinically relevant. Also, students feel that the quantity of material they are expected to learn is appropriate, with some believing the material is excessive and others believing it is insufficient. Of course, it is difficult to know whether student opinion is an accurate predictor of how well a student will be prepared for clinical practice, the main concern being that students do not yet know what the demands of clinical practice are. To better assess the effect of reduced quantity in anatomy education on medical student preparedness for residency, we surveyed two populations of alumni from the University of Michigan Medical School (UMMS) who are currently in their postgraduate training. The first population completed an older medical school curriculum (OC group) that included $\sim 175$ hours of gross anatomy instruction and 38 dissection laboratory sessions. In 2004, the gross anatomy curriculum was reduced to 136 hours of instruction and 32 dissection laboratory sessions; the second population of polled alumni completed this new curriculum (NC group). The anatomy course for 1st year medical students at UMMS is a clinically based course, with test questions written in a clinical case format, patient histories presented with each cadaver, an emphasis on framing the cadaver as the medical student's first patient, clinical cases included in the laboratory manual learning material, and a nolecture format that encourages self-reliance in learning. This emphasis on clinical anatomy did not change with the inception of the NC. The only two changes to 1 st year anatomy brought on by the NC were reduced quantity of anatomy instruction (no overt change in quality) and the elimination of separate grading for anatomy (OC students needed a passing grade in anatomy and other basic science disciplines, whereas NC students need only to pass each systems-based sequence; anatomy could be failed and the student still progress with a passing grade in the sequence). Effectively, the NC affords 1st year students the same quality of anatomy education but reduced quantity and reduced incentive to study. Fourth year anatomy electives provide UMMS students an opportunity to study via cadaver dissection areas of anatomy most pertinent to their chosen specialties, with a greater emphasis on anatomical considerations in clinical interventions. These electives also remained the same from the OC to the NC. By polling two populations of UMMS alumni across the curriculum change, we hope to shed more light on the effect of reduced quantity in anatomy curricula on medical student preparedness for postgraduate training.

\section{METHODS}

A five-question Likert scale survey was sent to the most recent four classes of UMMS medical school alumni to ensure that all respondents were currently in their residency training. Approximately 650 residents were sent the survey (an exact number is not known because the Medical College Alumni Society (MCAS) sent the survey on our behalf so as to keep alumni contact information confidential). Half of those residents polled were from the old curriculum group (OC) and the other half from the new curriculum group (NC). Survey responses were collected using Qualtrics survey software, version 15538, (Qualtrics Labs Inc., Provo, Utah), and data analysis was conducted using the SPSS statistical analysis program, version 12.0 (SPSS Inc., Chicago, IL). The data were first divided into $\mathrm{OC}$ and $\mathrm{NC}$ groups, and responses were compared using independent samples $T$-test. The data were then divided into surgical specialty (SS) and nonsurgical specialty (NS) groups, and responses were compared using independent samples T-test. Finally, the SS group and NS group were separated, and within each specialty group, the affect of curriculum (OC or NC) was compared, again via independent samples $T$-test. Specialties were designated as surgical or nonsurgical based on the American College of Surgeons listing of surgical specialties, which includes general surgery, neurosurgery, obstetrics and gynecology, ophthalmology, orthopedic surgery, otolaryngology, and plastic surgery.

The first three questions on the survey concern respondents' gross anatomy experience as a whole. We asked the following questions:

1. How well do you think your anatomy education in medical school prepared you for internship/residency $(1=\mathrm{It}$ did not prepare me at all, $10=$ It prepared me as much as possible)?

2. Do you think a more or less robust anatomy component in medical school would have better prepared you for internship/residency $(1=$ Much less robust, $5=$ Neither more nor less robust, $10=$ Much more robust)?

3. How important do you feel cadaver dissection was to your anatomy preparation for internship/residency $(1=$ Not at all important, $10=$ Extremely important)?

The last two questions on the survey were specifically about 4th year anatomy electives. Of those students who had taken a 4th year anatomy elective, we asked the following:

4. How effective was your 4th year anatomy elective in expanding your anatomy education $(1=$ Very ineffective, $10=$ Very effective)?

5. How effective was your 4th year anatomy elective in preparing you for internship/residency ( 1 = Very ineffective, 10 = Very effective)?

A space for comments was provided at the end of the survey.

\section{RESULTS}

One month after the survey was sent, responses had ceased coming in. One hundred and ten responses were received. Seventy-one respondents were OC and 39 respondents were NC, 47 respondents were SS and 63 respondents were NS 


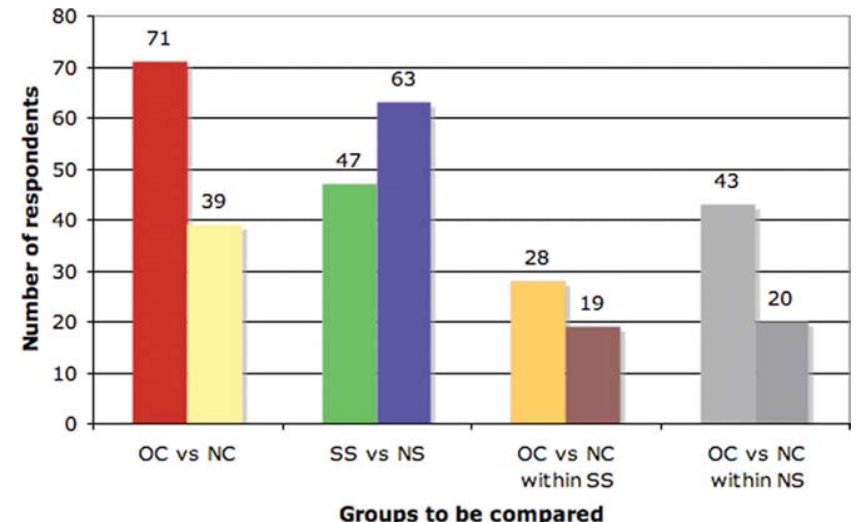

Figure 1.

$\mathrm{N}$-Values for Comparisons between Curriculum, Specialty, and Curriculum within Specialty Groups. OC = Old Curriculum, NC = New Curriculum, $\mathrm{SS}=$ Surgical Specialty, NS = Nonsurgical Specialty.

(see Fig. 1). Figure 2a summarizes the survey results for the entire respondent population and the main group analyses (OC vs. NC and SS vs. NS). As a whole population, the respondents felt that their anatomy education prepared them very well for residency (Question $1=7.55 / 10$ ). Respondents also reported as a whole that the robustness of their anatomy course was appropriate, with only a slight bias toward feeling that a more robust anatomy course would have better pre- pared them (Question $2=6.46 / 10$ ). Respondents felt that cadaver dissection was very important to their residency preparation (Question $3=7.23 / 10$ ). Of those students who took a 4th year anatomy elective, the overwhelming majority felt that this elective improved their anatomy education (Question $4=8.9 / 10$ ) and effectively prepared them for residency (Question $5=8.2 / 10$ ). A significant percentage of the respondents who did not take a 4th year elective commented at the end of the survey that they wish they had $(30 \%$ of SS group, $11 \%$ of all respondents, and $8 \%$ of NS group).

As seen in Figure 2a, no statistical difference existed between the $\mathrm{OC}$ and $\mathrm{NC}$ groups for any of the questions (Questions 1-5, P>0.05). Specialty (SS or NS) had no effect on respondent impressions of how well their anatomy education prepared them for postgraduate training (Question 1, $P>0.05)$. SS respondents felt more strongly than NS respondents that a more robust anatomy course would have better prepared them for residency (Question 2, $P=0.001$ ). SS respondents also felt more strongly than NS respondents about the importance of cadaver dissection to their preparation for residency (Question 3, $P=0.001$ ). Specialty did not have a significant effect on respondent impressions of 4th year anatomy electives and their effectiveness in expanding one's anatomy education (Question $4, P>0.05$ ) or preparing one for internship/residency (Question 5, $P>0.05$ ).

Figure $2 \mathrm{~b}$ shows the results of the subgroup analyses (OC vs. NC within SS and OC vs. NC within NS). Within the SS subgroup, effect of curriculum was insignificant for all questions (Questions 1-5, P>0.05). Within the NS subgroup, effect of curriculum was also insignificant for all questions (questions 1-5, $P>0.05$ ), though it approached significance for how well respondents felt their anatomy education pre-
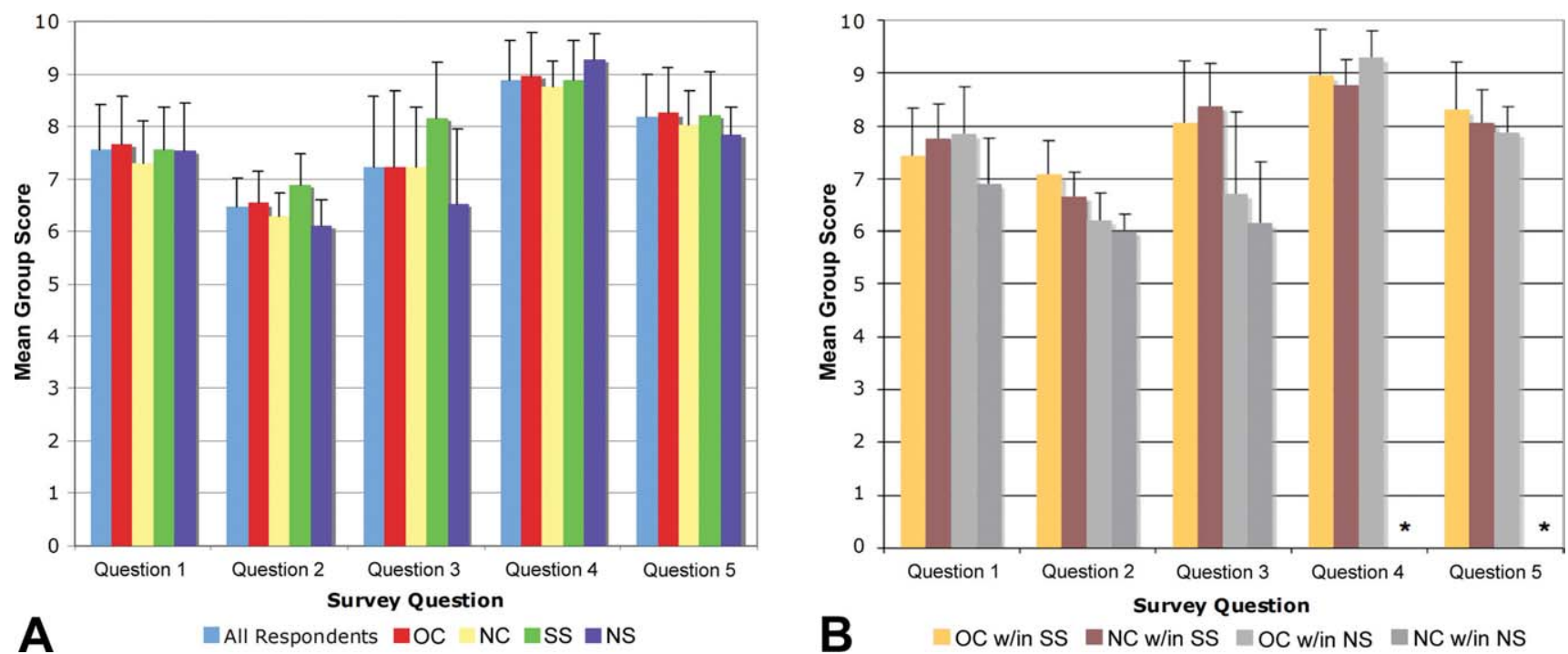

\section{Figure 2.}

Survey of Medical College Alumni from the University of Michigan Medical School Regarding Anatomy Curriculum. (A) Effect of curriculum on alumni respondents by surgical and nonsurgical Specialty; (B) Effect of curriculum on respondents within specialty groups. Question 1: How well do you think your anatomy education in medical school prepared you for internship/residency?; Question 2: Do you think a more or less robust anatomy component in medical school would have better prepared you for internship/residency?; Question 3: How important do you feel cadaver dissection was to your anatomy preparation for internship/residency?; Question 4: Please answer if you took a 4th year elective course in anatomy: How effective was your 4th year anatomy elective in expanding your anatomy education?; Question 5: Please answer if you took a 4th year elective course in anatomy: How effective was your 4th year anatomy elective in preparing you for internship/residency? Abbreviations: OC $=$ Old Curriculum, NC $=$ New Curriculum, SS = Surgical Specialty, NS = Nonsurgical Specialty, $*$ No score is reported-respondents in NS and NC group did not take a 4th year anatomy electives. 
pared them for residency (OC mean response was 7.84, NC mean response was $6.90, P=0.057)$. We received no respondents in the NC group who were also NS and took a 4th year elective, and so comparisons could not be made between curricula within the NS subgroup regarding 4th year electives.

\section{DISCUSSION}

Factors that must be taken into consideration when analyzing our results include our small response rate $(\sim 17 \%)$, and the possibility that those residents who chose to respond were more likely to be those with either a very positive or very negative experience in medical school gross anatomy. Because the UMMS MCAS keeps alumni contact information confidential, we needed their cooperation to send out our survey. MCAS was reluctant to "spam" the mailboxes of UMMS alumni, and so we were unable to send out more than one wave of e-mails requesting the completion of our survey. We recognize that our small response rate creates a potential source of bias in our results. However, as a pilot study, we accept that this survey may not provide conclusive results, hoping instead that it will motivate further, more comprehensive research on this topic.

No statistical difference was demonstrated between the OC and NC groups, neither as a whole nor as specialty subgroups, indicates that the 2004 reduction in anatomy instruction at UMMS was not so great as to change students perceived preparedness once in residency training, though it is interesting that both groups agreed that a slightly more robust anatomy curriculum would have better prepared them for residency. This may mean that although residents felt comfortably challenged by both the more and less intensive anatomy curricula (and so responded with equally positive scores), both groups felt as residents that they had not received enough anatomy preparation in medical school. In accordance with this observation is a study by Demars and coworkers that shows anesthesia residents perform better in complicated peripheral nerve blocks (specifically humeral blocks) after returning to the dissection laboratory to study the pertinent anatomy (Demars et al., 2010). Although these results did not hold true for simpler nerve blocks, other studies have shown that a return to cadaver dissection improves anesthesia residents' knowledge of anatomy, performance in nerve blocks, and professional practice thanks to safer technique (Urion et al., 2003; Lirk et al., 2005). These studies, along with our results, suggest two things: first, surgical residents (or those in anatomy intensive fields) benefit from a return to anatomical study via cadaver dissection and so residency programs should include a return to the anatomy laboratory, and second, medical school graduates are beginning their postgraduate medical training without the appropriate skill and knowledge base that should be afforded by undergraduate anatomy education.

Although it is not surprising that SS residents felt more strongly about the importance of cadaver dissection and the appropriate level of intensity of the anatomy curriculum than NS residents, it is important to note that this result indicates agreement between the surgical community and its residents that new graduates are beginning surgical residencies with inadequate preparation for their postgraduate study of anatomy. Although medical schools should not be responsible for preparing all students for careers in surgical fields, they do need to adequately prepare students for postgraduate study in any type of residency program. This means having an anatomy curriculum that builds a foundational knowledge of structure upon which future knowledge of anatomical considerations in clinical interventions can be placed. With this foundational knowledge in place, surgical residents will be adequately prepared for their postgraduate review of anatomy and return to the dissection laboratory, which has been shown by the data cited above (Urion et al., 2003; Lirk et al., 2005; Demars et al., 2010) to be beneficial to residents in anatomy intensive fields. Any medical school that wishes to continue attracting significant numbers of medical students interested in surgery, and graduating students well-prepared for a career in surgery, should therefore include cadaver dissection as a primary teaching method and should be very wary of reductions in gross anatomy instruction. Students, residents, medical school faculty, and postgraduate educators in surgery are in agreement that anatomy education in medical school needs to be bolstered.

Interestingly, the 4th year anatomy electives received the most positive responses. We believe this is because 4th year electives are the only opportunity for UMMS students to revisit focused anatomical study and cadaver dissection after gaining some clinical experience. That $30 \%$ of SS respondents who did not take a 4th year elective volunteered at the end of the survey that they wish they had suggests a real need for students to revisit gross anatomy toward the end of medical school, when students are least likely to remember the material and most likely to appreciate its clinical relevance. As Sugand and coworkers explain, "[anatomy] itself is relevant to the majority, if not all, healthcare specialties... Yet, graduates are expected to retain and rely on the knowledge from their preclinical years for the rest of their careers" (Sugand et al., 2010). The vertical integration of clinical anatomy throughout preclinical and clinical years of medical education is a historically proven model of anatomical instruction (Hildebrandt, 2010), and as our results indicate, its potential should be further realized in our modern medical school curricula.

As Figure $2 \mathrm{~b}$ demonstrates, OC respondents paradoxically reported a preference for a more robust anatomy curriculum than NC respondents regardless of specialty. One might have assumed that SS respondents who took the NC would report a stronger preference for a more robust anatomy curriculum than their counterparts in the OC owing to fact that they completed a much less rigorous anatomy curriculum. Likewise, one might expect the NS residents who took the more rigorous $\mathrm{OC}$ to report less desire for a more robust anatomy curriculum than their NC counterparts because they received far more anatomy. Surprisingly, the opposite trend is observed in our results. SS respondents in the OC report a higher preference for a more robust anatomy curriculum than their NC counterparts (question 2), and NS respondents in the OC report more positive scores than their NC counterparts for all the questions pertaining to 1 st year anatomy (questions 1-3). Although this trend does not represent a statistically significant difference in our data, we do believe it represents an effect that the curriculum change had on student perception of the importance of anatomy. By reducing the time devoted to anatomy and eliminating the separate grading of anatomy, the NC relegated anatomy in the 1 st year medical curriculum from a subject of prominent importance to a secondary topic that could be failed with little consequence. Perceptive to the diminished importance of anatomy in their 1st year studies, 
NC students were perhaps more likely to adopt the belief that anatomy is itself a less important topic in medicine. Such an effect on student opinion would produce the trend seen for question 2 in Figure $2 \mathrm{~b}$, in which $\mathrm{NC}$ respondents report less desire for a more robust anatomy curriculum than OC respondents, regardless of specialty. If this trend is valid, it provides evidence that cutting anatomy from the medical school curriculum may have long-term effects on how residents perceive the importance of anatomy to clinical practice, potentially resulting in a diminished level of anatomical knowledge, or a diminished desire to acquire anatomical knowledge, among newer generations of physicians.

In the current reformation of the modern medical school curriculum, anatomy has oftentimes been relegated to the sideline, being labeled by the most skeptical as a clinically useless exercise in memorization. But perhaps, it is a mistake to devalue anatomy as excessively factual and clinically irrelevant. After all, Moxham and Plaisant (2007) have recently shown that "contrary to the unquantified beliefs of those who are skeptical about the purpose and value of anatomy in an undergraduate medical curriculum, [students] at all stages of their medical course share with professional anatomists the view that anatomy is a very important subject for their clinical studies." Medical students and their anatomy professors are not alone in believing anatomy has great clinical relevance. Postgraduate educators also worry that the burden of factual anatomical information in medical school has been reduced to a clinically unsafe level. Concerns about the effect of anatomy's downward trend on medical student preparedness for clinical practice are manifest in nearly 30 years of reports from the surgical community about the importance of preserving anatomy education and dissection (Kaufman, 1997; Older, 2004; Shaffer, 2004; Sritharan, 2005; Turney, 2007). Although it may be easy to dismiss such concern as nostalgia within the surgical community for the "old-fashioned" curricula, these surgeons' concerns are substantiated by Ellis' (2002) report that the Medical Defense Union (MDU) experienced a sevenfold increase in legal claims associated with anatomical errors between 1995 and 2000, and that $32 \%$ of claims made to the MDU in general and vascular surgery were for "damage to underlying structures." Finally, the general public has even expressed concern with the declining role of anatomy in medical education, as a recent national television poll in the United Kingdom showed a high amount of apprehension over the extent of doctors' anatomical knowledge, with most people expressing preference for doctors who have had experience with genuine human dissection (Chowdhury et al., 2008). The concerns of the public are addressed by Sugand and coworkers who write, "the deconstruction of anatomy education and undermining [of] the crucial knowledge and skills gained from the course will inevitably lead to under-qualified educators for future generations as well as unsafe and incompetent doctors" (Sugand et al., 2010). The problem, then, seems not to be that professional anatomists, medical students, postgraduate educators, or the public are in disagreement over the need to maintain anatomy education (just the opposite is true), but that the logistical realities of creating a modern medical school curriculum force the medical administration's hand to keep creating more space in the preclinical years by cutting what has traditionally taken the most of student time and school resources. As anatomy education continues to take a smaller role in preclinical medical school curricula, we must wonder at what point do we impair students' ability to acquire new knowledge of the human body by failing to develop a firm understanding of human structure in which to place it.

In addressing this issue of anatomy preparation for medical students, Bergman and coworkers appropriately ask in their review article, "How much anatomy is enough?" The authors of this review attempt to answer this question by administering a case-based anatomy test using standards created by different groups of students and medical educators (Bergman et al., 2008). The authors conclude that although there is no agreement on what level of anatomical knowledge is appropriate, there is wide agreement among students and educators that students' anatomical knowledge is unsatisfactory. The authors further report that good test performances by students seem to depend not on a school's didactic approach to teaching, but on total teaching time for anatomy, teaching in clinical context, and revisiting anatomy concepts over the course of the curriculum (vertical integration). This should come as no surprise considering that these three approaches to anatomical education are the same principles touted by the great anatomists that made anatomical education in the United States a world-class endeavor over 100 years ago (Hildebrandt, 2010). The reality of our exponentially expanding medical knowledge is that modern medical school curricula will necessarily be focused on foundational topics that enable life-long learning and effective clinical practice. Although anatomy may no longer comprise the majority of the medical school curriculum that it once did, this does not mean that it cannot succeed in creating a foundational knowledge of structure for students to place future knowledge of function. In their study of undergraduate perspectives of anatomy, Mitchell and Batty (2009) make a reasonable argument that although cases can be made for reinvigorating undergraduate anatomy education, the modern medical school curriculum is here to stay and it will necessarily continue to evolve. Therefore, to achieve our ultimate goal of providing medical students a foundation for future learning and a platform for safe practice as an intern, anatomy education must be vertically integrated, using student encounters in outpatient clinics, operating theaters, and at the bedside to teach anatomy.

\section{CONCLUSIONS}

Our results support Mitchell and Batty's (2009) claim by showing how successful and popular clinical anatomy electives have been among 4th year students at UMMS. Finally, by vertically integrating anatomy into the modern medical school curriculum, we can also achieve more total curriculum time devoted to anatomy and greater clinical relevance, thereby continuing to implement the three historically proven principles of anatomy education that Bergman et al. (2008) show to still be effective. In doing so, we choose to evolve with the modern medical curriculum without sacrificing our historically most successful methods of anatomical instruction.

\section{ACKNOWLEDGMENT}

The authors would like to thank all of the University of Michigan Medical School alumni who responded to this survey. 


\section{NOTES ON CONTRIBUTORS}

MICHAEL A. BOHL, B.A., is a second year medical student at the University of Michigan Medical School, Ann Arbor, Michigan. He also works as an organ perfusion technician in the University of Michigan Transplant Center.

THOMAS R. GEST, Ph.D., is an associate professor of anatomy and Director of the Anatomical Donations Program at the University of Michigan Medical School, Division of Anatomical Sciences, Ann Arbor, Michigan. He teaches gross anatomy, applied clinical anatomy, and yoga to medical students.

\section{LITERATURE CITED}

Bergman EM, Prince KJ, Drukker J, van der Vleuten CP, Scherpbier AJ. 2008. How much anatomy is enough? Anat Sci Educ 1:184-188.

Chowdhury R, Wilson ID, Oeppen RS. 2008. The departments of radiology and anatomy: New symbiotic relations? Clin Radiol 63:918-920.

Demars N, Compère V, Duparc F, Fourdrinier V, Dureuil B. 2010. Contribution of the anatomy laboratory to the practical training of residents in regional anesthesia. Surg Radiol Anat 32:69-73.

Drake RL, McBride JM, Lachman N, Pawlina W. 2009. Medical education in the anatomical sciences: The winds of change continue to blow. Anat Sci Educ 2:253-259.

Ellis H. 2002. Medico-legal litigation and its links with surgical anatomy. Surgery 20:i-ii.

Gartner LP. 2003. Anatomical sciences in the allopathic medical school curriculum in the United States between 1967-2001. Clin Anat 16:434-439.

Gogalniceanu P, Palman J, Madani H, Sheena Y, Birch W, Paraskeva P, Douek M. 2010. Traditional undergraduate anatomy education-A contemporary taboo? ANZ J Surg 80:6-7.
Hildebrandt S. 2010. Lessons to be learned from the history of anatomical teaching in the United States: The example of the University of Michigan. Anat Sci Educ 3:202-212.

Holla SJ, Ramachandran K, Isaac B, Koshy S. 2009. Anatomy education in a changing medical curriculum in India: Medical student feedback on duration and emphasis of gross anatomy teaching. Anat Sci Educ 2:179-183.

Kaufman MH. 1997. Anatomy training for surgeons-A personal viewpoint. J R Coll Surg Edinb 42:215-216.

Leong SK. 1999. Back to basics. Clin Anat 12:422-426.

Lirk P, Colvin JM, Biebl M, Mitterschiffthaler G, Moser PL, Lorenz IH, Kolbitsch C. 2005. Evaluation of a cadaver workshop for education in regional anesthesia. Anaesthetist 54:327-332.

Mitchell R, Batty L. 2009. Undergraduate perspectives on the teaching and learning of anatomy. ANZ J Surg 79:118-121.

Moxham BJ, Plaisant O. 2007. Perception of medical students towards the clinical relevance of anatomy. Clin Anat 20:560-564.

Older J. 2004. Anatomy: A must for teaching the next generation. Surgeon 2:79-90.

Patel KM, Moxham BJ. 2008. The relationships between learning outcomes and methods of teaching anatomy as perceived by professional anatomists. Clin Anat 21:182-189.

Shaffer K. 2004. Teaching anatomy in the digital world. N Engl J Med 351:1279-1281.

Sritharan K. 2005. The rise and fall of anatomy. BMJ Careers 330:255-256.

Sugand K, Abrahams P, Khurana A. 2010. The anatomy of anatomy: A review for its modernization. Anat Sci Educ 3:83-93.

Turney BW. 2007. Anatomy in a modern medical curriculum. Ann R Coll Surg Engl 89:104-107.

Urion L, Mekler G, Marchand G, Heck M, Braun M, Dautel G, Mertes PM, Laxenaire MC, Bouaziz H. 2003. Impact of a continuous medical education on regional anaesthesia practice. Ann Fr Anesth Reanim 22:379-386. 